\title{
A colloquio con Antonio Porta
}

\section{John Picchione}

Occasione di questa intervista ad Antonio Porta è stata la sua partecipazione al World Poetry Festival svoltosi a Toronto, presso Harbourfront, nel maggio scorso. Per chiarire alcune dichiarazioni relative soprattutto alle varie fasi dell'opera di Porta, forniamo delle brevi notizie bio-bibliografiche.

Antonio Porta è nato nel 1935. Risiede a Milano. Nel 1961 ha contribuito alla formazione de I Novissimi. Poesie per gli anni '60 a cura di Alfredo Giuliani. Redattore delle riviste Il Verri e Malebolge, è stato tra i fondatori del periodico Quindici ed ha partecipato ai lavori del Gruppo 63. È stato critico letterario de Il Corriere della Sera, de Il Giorno, ed ha collaborato a Tuttolibri. Attualmente fa parte della redazione di Alfabeta e de La Gola. Si è anche occupato di poesia visiva ed ha partecipato ad alcune mostre a Padova, Milano, Roma e Londra. Sue poesie sono state tradotte in varie lingue: inglese, francese, tedesco, spagnolo, ungherese, olandese, cecoslovacco. La sua prima raccolta di poesie, La palpebra rovesciata (Azimuth) esce nel 1960. Seguono Zero (ed. numerata, 1963); Aprire (Scheiwiller, 1964); I rapporti (Feltrinelli, 1965); Cara (Feltrinelli, 1969); Metropolis (Feltrinelli, 1971); Weekend (Cooperativa Scrittori, 1974). Nel 1977 pubblica la raccolta completa delle sue poesie dal 1958 al 1975 con il titolo Quanto ho da dirvi (Feltrinelli). Nel 1980 esce un nuovo libro di poesie Passi passaggi (Mondadori) e nel 1984 con Invasioni (Mondadori) vince il premio Viareggio. Nel campo della narrativa ha pubblicato due romanzi Partita (Feltrinelli, 1967), (ristampato nel 1978 da Garzanti), Il re del magazzino (Mondadori 1978), ed un volume di racconti Se fosse tutto un tradimento (Guanda, 1981). Ha scritto anche un'opera teatrale, La presa di potere di Ivan lo sciocco (Einaudi, 1974). Delle sue traduzioni ci limitiamo a ricordare La stangata persiana di Plauto (Corpo 10, 1985). Ha curato tra l'altro l'antologia Poesia degli anni settanta (Feltrinelli, 1979). Ultimamente ha pub- 
blicato una raccolta antologica delle sue poesie con il titolo $\mathrm{Nel}$ fare poesia (Sansoni, 1985).

D. - La tua poesia è sempre stata caratterizzata da una continua sperimentazione e quindi lontana da pericolosi approdi definitivi. Comunque nelle tue ultime prove, penso a Invasioni in particolare, la presenza di un linguaggio allusivo, analogico, costruito su continui spostamenti metaforici, rivela una profonda distanza rispetto alla tua prima produzione. Cosa ha sollecitato tale svolta?

R.-Penso di poter addirittura dare una data a questa svolta perché mi ricordo abbastanza bene il periodo: è il 1976. L'ultima serie di poesie che ho scritto prima di questa svolta è "Passeggero" che ho pubblicato in Quanto ho da dirvi nella sezione inedita ed erano le uniche poesie che ho scritto nel 1975. Ad un certo punto mi sono reso conto che tutti i progetti che avevo portato a termine, compreso appunto "Passeggero," mi portavano in una situazione senza uscita: o potevo diventare ripetitivo, allora diventavo, come dire, un manierista di me stesso, oppure dovevo assolutamente trovare una via d'uscita. La via d'uscita può essere definita come un progetto di comunicazione poetica, ecco perché ho cominciato a scrivere le brevi lettere. "Brevi lettere" era il primo titolo di questa serie che ho cominciato proprio nel 1976. Con le brevi lettere mi sono rivolto direttamente ai miei figli che allora avevano circa 17-18 anni. I miei figli desideravano comunicare con me, come lo desiderano tutt'ora, e trovavano la mia poesia poco comunicativa, troppo distante, diciamo troppo formale. Allora ho pensato che la poesia doveva tentare la strada della comunicazione come anche in altre epoche della sua storia, e quindi ho cominciato questo esperimento di poesia comunicativa che naturalmente ha i suoi pericoli, un pericolo maggiore è la banalità, cioè di dire cose che si sanno già prima. Non so se ho evitato fino in fondo tali pericoli, spetta ai lettori giudicare, però ne sono cosciente. Tuttavia, credo che sia un rischio da correre. Del resto, questo nuovo progetto di poesia ha avuto subito un riscontro positivo. Per esempio, al Poetry International, il festival di Rotterdam, la risposta è stata altamente positiva e questo $\mathrm{mi}$ ha incoraggiato. Io credo che la poesia debba parlare ad un pubblico, non può essere una costruzione fine a se stessa, la poesia parla a qualcuno, non c'è niente da fare. Questo qualcuno può essere anche un fantasma, poniamo, metafisico, il famoso "tu" montaliano, però c'è questo "tu." Voglio dire questo: non ho scrtto piú brevi lettere dopo il 1980 perché il progetto 
non ha avuto piú seguito, non mi sono piú sentito di continuarlo. Vorrei precisare che, come si vede anche nel mio ultimo libro di poesia, Nel fare poesia, un'antologia che contiene anche inediti, io ho sempre lavorato a progetti diversi o diversificati perché credo che un poeta non sia l'autore di un solo libro, non è vero assolutamente. Un artista lavora a diversi tipi di espressioni e di forme, a meno che poi, ad un certo punto nella sua vita, trovi la forma finale e con quella magari va avanti fino alla morte. Questo non mi è ancora successo e forse non è necessario che succeda. Pound che era il grande sperimentatore che sappiamo, ad un certo punto ha trovato la forma dei Cantos che non ha piú abbandonato. Contemporaneamente, dunque, alla forma, chiamiamola cosí, comunicativa delle brevi lettere, ho scritto altre cose: per esempio, ho ritentato la strada dell'immagine forte nella serie "New York," poesie che cercano, trovano la comunicazione nell'immagine. Poi ho invece ripreso la forma delle brevi lettere nel diario "Come può un poeta essere amato?" Quella è una forma diaristica, ma anche il diario ha una sua forma: molto spesso si parla di poesia diaristica senza specificare che anche la forma diario è appunto una forma. È essenziale dire questo, altrimenti si cade nell'equivoco di scrivere della poesia di confessione. Cosí come le poesie brevi di Invasioni, la serie che dà il titolo alla raccolta, sono una forma che ricorda lo haiku giapponese nel senso che ci sono dentro spostamenti di piani linguistici molto forti, e questo spesso si perde nella traduzione inglese, non so se per colpa dei traduttori o perché piú difficile in inglese. È molto arduo cogliere tutti questi spostamenti semantici per uno che non conosca l'italiano come lo conosce l'autore.

D.-A proposito del tuo ultimo libro, Nel fare poesia, in alcuni commenti introduttivi poni molta enfasi sulla poesia intesa come trasfigurazione e come esperienza del prelinguistico. Cosa è avvenuto alla poetica della poesia "in re"?

R.-Ho parlato di espressione del prelinguistico, cioè dare forma al prelinguistico. Trasfigurazione invece vuol dire, sostanzialmente, una sorta di riscatto, formale appunto, dell'oggetto, e quindi l'inserimento del sistema degli oggetti ripresi dal sistema percettivo in una forma che sia trasfigurata, cioè a dire, illuminata come dall'interno. Evidentemente, rispetto alla poesia "in re" è un salto notevole perché, anche da un punto di vista teorico, la poesia "in re" era una poesia essenzialmente tragica in quanto vedeva l'immutabilità della cosa, la prevalenza della cosa sull'uomo. Qui, invece, direi che voglio vedere la cosa come rinasce 
dentro il linguaggio. Questo è il punto. Sia ben chiaro, quando parlo di trasfigurazione non parlo di esperienza religiosa, ma di un'esperenza linguistica, è il linguaggio che riflette se stesso. Io non voglio rispecchiare la realtà, questo non è lo scopo della mia poesia, ma vedere come la realtà e il linguaggio interagiscono e quindi come il linguaggio rispecchiandosi, rispecchiando se stesso, può trasfigurare, cambiare forma, figura all'esperienza. È questo il senso che do a questa parola.

D.-Quindi la poesia nascerebbe di nuovo all'interno di se stessa?

R.-Questo è l'eterno problema. Ci sono sempre due punti di partenza per la poesia, lo diceva anche Valéry, credo che lo abbiano detto tutti i poeti nella storia: puoi partire da una intenzione formale, - primo verso diceva Rilke, poi gli altri seguiranno - oppure dalla volontà di esprimere quello che sta prima della lingua, appunto dare forma al prelinguistico come dicevo io. Però questo non significa dimenticare il fatto che la poesia è un'esperienza linguistica, cioè di linguaggio. Non è una posizione conciliante la mia, bisogna semplicemente definire con precisione i momenti del lavoro poetico. Io lo chiamo sempre lavoro poetico perché credo che sia anche un lavoro artigianale, che sia una conoscenza profonda della lingua e dei suoi possibili meccanismi. Naturalmente, non tutti questi meccanismi sono controllabili da parte di un autore; di qui nasce il problema dell'interpretazione, però, per lo meno, il progetto può essere spiegato.

D.-Hai partecipato al convegno di Palermo dell' '84 promosso da Alfabeta. A tuo avviso, è stato un tentativo di riprendere il discorso del Gruppo 63 o si è trattato piuttosto di un abbandono totale delle premesse letterarie e culturali degli anni '60?

R.-Nessuna intenzione di riprendere i lavori del Gruppo $63 \mathrm{ma}$ piuttosto di ricominciare d'accapo per rifarci e rifare una domanda fondamentale: che cos'è la letteratura? Domanda che sembra ingenua invece è pesantissima perché vuol dire: che cos'è la letteratura nel mondo di oggi, la letteratura nel mondo della tecnologia, dei mass media, dell'industria culturale? Cioè, la letteratura è ancora fonte di conoscenza? Trasmette immagini? Trasfigura l'esperienza? Inventa il linguaggio? Si possono fare infinite domande dentro questa domanda. Allora, se oggi ci chiediamo, in fondo che cos'è la letteratura, di qui può nascere la domanda successiva: come si costruisce o come si produce un'opera letteraria? Infatti, abbiamo organizzato a Roma nel dicembre del 1985 
un convegno italo-francese promosso dalla Quinzaine littéraire e da Alfabeta, intitolato "Io parlo di un certo mio libro," dove ciascuno ha spiegato come ha lavorato a un certo suo libro, che è la domanda successiva a quella fondamentale la quale in Italia, per esempio, faceva sorridere gli accademici. Secondo loro non ha senso domandarselo. Invece, per uno che lavora fino in fondo e crede nella verità della scrittura è una domanda essenziale, altrimenti diventa accademia. Cioè, sanno tutti cos'è la letteratura nell'accademia, ma è fuori dell'accademia che occorre porsi la domanda.

D.-In ogni caso, quali linee programmatiche o teoriche di maggiore rilievo sono emerse, a tuo avviso, dal convegno? Quali sono le differenze rispetto alle posizioni degli anni '60?

R.-Non è che ci siano linee teoriche particolarmente nuove. Diciamo che c'è un postmoderno, molto discusso e molto avversato anche perché arriva fino al Neoclassicismo, che ammette una conbinazione degli stili e quindi una liberazione delle forme per trovare una forma piú combinatoria o piú collagistica. Dall'altra parte, c'è una linea neoespressionista alla Leonetti e alla Volponi. Altri sostengono l'esperienza materialistica del linguaggio, espressione che francamente non so bene cosa significhi; forse vogliono dire la filosofia materialistica ripresa dal linguaggio della poesia. Comunque, nessuno è riuscito a spiegarmelo, nemmeno Leonetti che pure è inscritto in questo sistema della scrittura materialistica. Forse intendono scrittura materialistica versus scrittura spiritualistica, perché c'è stato in Italia un certo ritorno all'orfismo e all'ermetismo. Poi c'è la posizione di Sanguineti che è ancora bloccata, magari a ragion veduta, dentro la critica feroce della stessa letteratura: lui parla di boicottare la letteratura, di scrivere "male," vale a dire di sapere bene come scrivere "male." Questo è il paradosso di Sanguineti. In realtà, poi, lui scrive benissimo. Infatti, nel suo ultimo libro, Novissimum testamentum, c'è un poemetto, tutto in ottave, di un'abilità sconvolgente da un punto di vista tecnico. Si tratta di ottave degradate in un certo senso, però fatte benissimo. Inoltre, c'è la posizione di Franco Fortini che è piú vicina a quella di Sanguineti: il sabotaggio della letteratura come sovrastruttura borghese, come inganno della classe dominante. Comunque, tutte queste posizioni convivono. Il problema è grosso perché non ci sono piú delle certezze ideologiche, dei sentieri tracciati, qui siamo un po' nell'epoca dei sentieri interrotti, quelli di cui parlava Heidegger. Infatti, c'è anche un ritorno a Heidegger da questo punto di vista, senza metafisica se si 
vuole. Quindi ci sono tutte queste possibilità. Per trovare il proprio stile dentro questa giuı.gla, ognuno si prende le proprie responsabilità, prende il suo machete e si trova la strada.

D.-A proposito di sistemi filosofici, il pensiero debole sta attirando molta attenzione anche da parte di vari letterati. Quali sono le tue reazioni come poeta e come intellettuale?

R.-Come ha detto Rovatti, un filosofo che ha partecipato al volume che ha innescato la moda, il pensiero debole è un' infelice metafora, il che significa che vuol dire tutto o niente. In sostanza, a leggere Vattimo, un filosofo di primaria importanza in Italia, il pensiero debole è un pensiero non rivoluzionario, nemmeno forse riformatore, ma è un pensiero che trova il suo percorso in quello che c'è già, nello status quo, nella società, e ne segue gli sviluppi sostanzialmente del cambiamento cosí come la società lo esprime. Al contrario, il pensiero "forte" dovrebbe essere il pensiero che magari rifiuta integralmente questa società e progetta un nuovo modo di essere o di pensare. Ecco perché Vattimo è accusato di essere in fondo un filosofo del neocapitalismo, del capitalismo avanzato o chiamiamolo del terziario. Devo dire che in questa accusa c'è un fondamento di verità, perché il pensiero debole in fondo vorrebbe dire convivere abilmente con tutto. Del resto, comunque, anche il contrasto cercato a tutti i costi sembra forte ma è perdente. Oggi la posizione dell'artista o del filosofo all'interno della rinascita capitalistica è molto complicata.

D.-Quindi siamo molto lontani rispetto alle prospettive di avversione al capitalismo degli anni '60?

R.-Direi di sí. È proprio questo l'atteggiamento che è profondamente cambiato in tutta la sinistra europea, ma anche nel partito comunista italiano. C'è un'accettazione fondamentale della legge del mercato e quindi del capitalismo, proprio perché si è scoperto una cosa, e lo dico anch'io che sono pure un uomo di sinistra, non mi vergogno a dirlo: si è scoperto che non puoi distruggere la ricchezza per ridistribuirla; si tratta di trovare un sistema riformista, riformista come il sistema della socialdemocrazia europea che ha cercato una via di mezzo tra l'accumulazione della ricchezza e la sua ridistribuzione. L'errore gravissimo che hanno fatto nel socialismo cosiddetto reale è che hanno distrutto la ricchezza, dopo di che non c'era piú nulla da distribuire a nessuno. Infatti, l'esperienza ungherese ce lo insegna. Hanno ricominciato ad accumulare ricchezza, a creare e a produrre ricchezza, ed iniziano a stare meglio, senza negare i principi fon- 
damentali del socialismo, di giustizia e di uguaglianza. In questo senso, sí, il pensiero è debole perché naviga in acque ambigue se vogliamo. lo penso questo personalmente, l'esperienza del Canada e degli Stati Uniti me lo sta insegnando: noi subiamo, da una parte, la seduzione del capitale, non c'è niente da fare il capitale offre bellezza, offre felicità, lo ha sempre fatto. Il commercio insegue e facilita i desideri dell'uomo, e tra questi c'è anche il desiderio della bellezza e dello star bene. Dall'altra parte, il dominio assoluto del mondo della finanza, - che del resto però dura dal '500 - non è una grande novità. Qui in Canada e negli Stati Uniti lo si vede in maniera molto piú chiara per una semplice ragione: le grandi banche hanno tutte il loro totem araldico, come l'avevano gli indiani, questi grandi grattacieli stupendi. In Italia si vede meno, è un paese povero rispetto al Nord America, e quindi, la ricchezza è piú nascosta, piú strisciante, piú segreta.

D.-Torniamo comunque alla poesia. Dopo i poeti della parola innamorata non mi sembra ci siano stati nuovi gruppi con tendenze innovative sostanziali, In ogni caso, come giudichi la situazione della poesia oggi in Italia?

R.-La parola innamorata è stato un tentativo, in parte anche felice, di restituire un valore alla teoria, alla poetica. Ora la situazione, secondo me, non è piú, come dicevo prima, di gruppi, ma di individui che pur consapevoli di tutti i problemi, tracciano un loro percorso ma senza collegamenti. Forse si potrà vedere fra alcuni anni qualcosa che unifica le varie esperienze, ma non è una volontà antecedente, casomai è un disegno che si vedrà dopo.

D.-È questo sintomo di frammentarietà culturale o di isolamento dei singoli scrittori?

R.-Sí è vero, perché c'è certamente piú isolamento rispetto ai tempi del Gruppo 63, quando c'era piú interazione. Adesso si cerca di trovare dei punti di riferimento che però non sono teorici, sono piuttosto dei punti d'appoggio. Alfabeta, ad esempio, svolge una funzione di punto d'approdo ma non di un lavoro propositivo. $\grave{E}$ un punto di convivenza tra persone che la pensano anche molto diversamente.

D.-Abitare il linguaggio, stare dentro il linguaggio, è per te la premessa fondamentale del fare poetico. Perché e quali rapporti col linguaggio sono oggi necessari per un poeta? 
R.-Un poeta si nutre di linguaggi. Abitare il linguaggio non tanto, non solo per lo meno nel senso che Heidegger ha dato a questa parola; per lui il linguaggio è in fondo la "casa dell'essere." Lasciamo stare il problema dell'essere, - l'ho scritto anche in quel poemetto abbastanza divertente "Balene delfini bambini" vediamo invece l'uomo nella sua realtà antropologica e culturale. Secondo me, l'uomo è un animale linguistico; il linguaggio, quindi, è la sua "casa," è la "casa" dell'uomo, non dell'essere. Io non credo all'essere originario, assolutamente. L'essere cosí come lo ha inteso Heidegger non esiste. Il linguaggio nutre il poeta e il poeta se ne serve per fare poesia. Anzi, io penso che un poeta debba essere onnivoro, cioè mangiare tutti i linguaggi, poi non si sa quando li userà. Ad esempio, ho tradotto in versi Persa di Plauto usando tutti i linguaggi comici che avevo introiettato nella mia vita e che non avevo mai usato a quel livello, eppure li conoscevo. In questo senso la traduzione è stata giudicata postmoderna, perché ha usato sincronicamente e sincreticamente l'antico e il moderno, ha saltato tutte le diacronie possibili e ha attualizzato Plauto, pur rimanendogli perfettamente fedele. Infatti, Chiarini che ha studiato a lungo Persa, mi ha fatto un grosso complimento dicendomi che proprio cosí l'avrebbe scritto Plauto oggi.

D.-Hai nuovi progetti? Ti cimenterai di nuovo con un romanzo?

R.-Io ho molti nuovi progetti: un romanzo per cui ho un' idea già molto precisa, due lavori teatrali, delle poesie che sto scrivendo, un poemetto. . . . Progetti ne ho, credo per altri cent'anni, però penso che morirò prima di finirli.

York University 